\title{
Progressive restoration of spinal sagittal balance after surgical correction of lumbosacral spondylolisthesis before skeletal maturity
}

\author{
Diala Thomas, MD, MSc, ${ }^{1}$ Manon Bachy, MD, MSc, ${ }^{1}$ Aurélien Courvoisier, MD, PhD, ${ }^{2}$ \\ Arnaud Dubory, MD, MSc, ${ }^{1}$ Houssam Bouloussa, MD, ${ }^{1}$ and Raphaël Vialle, MD, PhD'1 \\ 1 Université Pierre et Marie Curie-Paris and Department of Paediatric Orthopaedics, Armand Trousseau Hospital, Paris; and \\ 2University Clinic of Paediatric Surgery, Paediatric Orthopaedics, Hôpital Couple-Enfant, Grenoble University Hospital, \\ Joseph-Fourier University, Grenoble, France
}

\begin{abstract}
OBJECT Spinopelvic alignment is crucial in assessing an energy-efficient posture in both normal and disease states, such as high-displacement developmental spondylolisthesis (HDDS). The overall effect in patients with HDDS who have undergone local surgical correction of lumbosacral imbalance for the global correction of spinal balance remains unclear. This paper reports the progressive spontaneous improvement of global sagittal balance following surgical correction of lumbosacral imbalance in patients with HDDS.

METHODS The records of 15 patients with HDDS who underwent surgery between 2005 and 2010 were reviewed. The treatment consisted of $\mathrm{L} 4$-sacrum reduction and fusion via a posterior approach, resulting in complete correction of lumbosacral kyphosis. Preoperative, 6-month postoperative, and final follow-up postoperative angular measurements were taken from full-spine lateral radiographs obtained with the patient in a standard standing position. Radiographic measurements included pelvic incidence, sacral slope, lumbar lordosis, and thoracic kyphosis. The degree of lumbosacral kyphosis was evaluated by the lumbosacral angle. Because of the small number of patients, nonparametric tests were considered for data analysis.
\end{abstract}

RESULTS Preoperative lumbosacral kyphosis and L- 5 anterior slip were corrected by instrumentation. Transient neurological complications were noted in 5 patients. Statistical analysis showed a significant increase of thoracic kyphosis on 6 -month postoperative and final follow-up radiographs $(p<0.001)$. A statistically significant decrease of lumbar lordosis was noted between preoperative and 6 -month control radiographs $(p<0.001)$ and between preoperative and final followup radiographs $(p<0.001)$.

CONCLUSIONS Based on the authors' observations, this technique resulted in an effective reduction of $\mathrm{L}-5$ anterior slip and significant reduction of lumbosacral kyphosis (from $69.8^{\circ}$ to $105.13^{\circ}$ ). Due to complete reduction of lumbosacral kyphosis and anterior trunk displacement associated with L-5 anterior slipping, lumbar lordosis progressively decreased and thoracic kyphosis progressively increased postoperatively. Adjusting the sagittal trunk balance produced not only pelvic anteversion, but also reciprocal adjustment of lumbar lordosis and thoracic kyphosis, creating a satisfactory level of compensated global sagittal balance.

http://thejns.org/doi/abs/10.3171/2014.9.SPINE1412

KEY WORDS lumbosacral spondylolisthesis; spinal sagittal balance; lumbosacral kyphosis; thoracic kyphosis; lumbar lordosis; pelvic incidence

$\mathrm{T}$ HE relation of the pelvis to the spine has previously been overlooked as a factor in sagittal balance. Studies have shown that certain structural features of the pelvis modulate and largely determine the degree of standing lumbar lordosis, as well as the sagittal pelvic alignment and spinopelvic balance. ${ }^{4,5,15,27}$ These relationships have been documented in adult volunteers ${ }^{4,5,7,15,29}$ and in patients with spondylolisthesis. ${ }^{6,10,11,16,22,28}$ Spinopelvic alignment is now recognized as crucial in the assessment of an energy-efficient posture in both normal 
and disease states. Legaye et al ${ }^{15}$ have described pelvic incidence as a specific and constant pelvic anatomical parameter that determines pelvic orientation, as well as the degree of lumbar lordosis. Recent studies ${ }^{13,14,17-19,26,28}$ have examined the correlation between an increased pelvic incidence and L5-S1 isthmic spondylolisthesis in adults and adolescents. In a spondylolisthesis patient with elevated pelvic incidence, the spine adapts with increased lumbar lordosis. ${ }^{28}$ To prevent or limit sagittal imbalance, the spine may also compensate with increased pelvic tilt or pelvic retroversion in an attempt to maintain an upright posture. ${ }^{26}$

While many different surgical strategies to correct high-grade lumbosacral spondylolisthesis have been reported, the overall effect of local surgical correction of lumbosacral imbalance on global correction of spinal balance remains unclear. ${ }^{8}$ We present a cohort of adolescents with high-grade lumbosacral spondylolisthesis and severe lumbosacral kyphosis, in which surgical correction of lumbosacral imbalance resulted in progressive spontaneous improvement of global sagittal balance.

\section{Methods}

The records of 15 consecutive patients who underwent surgical treatment between 2005 and 2010 for high-displacement developmental spondylolisthesis (HDDS) were reviewed. For each patient, clinical evolution from diagnosis to the final follow-up was noted.

The treatment consisted of L4-sacrum reduction and fusion via a posterior approach (Fig. 1). Each patient was placed prone under general anesthesia. No intraoperative electrophysiological monitoring was used. After removal of the posterior arch of L-4, L-5, and S-1, extensive decompression of the L-4, L-5, and S-1 nerve roots was performed. At the same time, 2 pedicular screws (Xia Spinal System, Stryker Spine) were inserted through the L-4 and L-5 vertebrae. Two S-1 ascending multiplanar pedicular screws were then inserted and pushed anteriorly into the posterior part of S-1. Screw placement was controlled intraoperatively by fluoroscopy. L4-5 and L5-S1 discectomies were performed, and anterior interbody fusion was achieved using 2 Trabecular Metal TM-500 Implants (posterior lumbar interbody fusion, Zimmer France) in
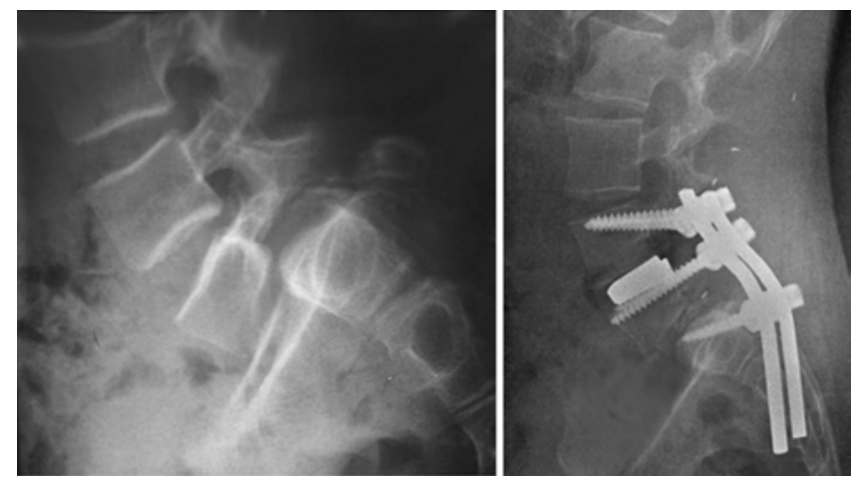

FIG. 1. Preoperative (left) and postoperative (right) lateral radiographs obtained in a 14-year-old patient with HDDS. Note the complete correction of lumbosacral kyphosis and the almost complete reduction of L-5 anterior slip.
L4-5 and cancellous bone insertion in L5-S1. Reduction was then obtained by inserting intrasacral Vitallium rods (Xia Spinal System, Stryker Spine; 6-mm-diameter rods for patients weighing more than $35 \mathrm{~kg}$ and $4.5-\mathrm{mm}$-diameter rods for smaller patients). The rod was pushed down through the S-2 vertebral body and toward the anterior part of the S-2 vertebral body, and it was then contoured and progressively locked in by pedicular screws at L-4 and L-5 by means of 2 persuaders. Immediately postoperatively, patients were placed supine with their knees flexed. To prevent nerve root stretching, the knees underwent progressive extension during the first 5 days, and the standing position was achieved on Day 8. No additional bracing was needed. Full activities, including sports, were allowed at Month 4.

Preoperative, 6-month postoperative, and final followup postoperative angular measurements were taken from full-spine lateral radiographs obtained in a standard standing position..$^{27}$ The subject assumed a comfortable standing position, with the knees fully extended and the upper limbs raised horizontally forward at $45^{\circ}$ of flexion at the shoulder, resting on 2 arm supports. The central ray was centered on the T-12 vertebra, and the film was exposed during inspiration. These radiographs visualized the complete axial skeleton between the external auditory ducts and superior third of the femurs.

Radiographic measurements included the classic angular parameters for assessing sagittal spinal balance: pelvic incidence, sacral slope, lumbar lordosis between the lower endplate of L-1 and the upper endplate of L-5, and thoracic kyphosis between the lower endplate of T-4 and the upper endplate of T-12. ${ }^{26-29}$ The degree of L-5 anterior slip was measured, and grades were defined according to Meyerding's classification. ${ }^{21}$ We considered Grade 5 to be complete spondyloptosis of L-5. Lumbosacral kyphosis was evaluated according to the lumbosacral angle, which is the reciprocal of the slip angle described by Boxall and colleagues. ${ }^{3,26}$

\section{Statistical Analysis}

Measurements were performed by 2 senior surgeons (D.T. and R.V.) using the same computer-assisted technique (Kodak Carestream PACS) and were stored in a computer database. The data were analyzed using SPSS software (version 21.0, SPSS Inc.). Because of the small number of patients, nonparametric tests were considered for data analysis. A p value for Wilcoxon's rank test was considered significant if $<0.05$.

\section{Results}

A total of 15 patients with developmental lumbosacral spondylolisthesis were enrolled in the study. The patient population included 11 females and 4 males, ranging in age from 8 to 16.8 years (mean 13.2 years). The mean preoperative percentage of L-5 anterior slip was 73\% (range 50\%-100\%). According to Meyerding's classification, 9 patients had a Grade 3 slip, 5 had a Grade 4 slip, and 1 patient had complete (Grade 5) spondyloptosis. Preoperative lumbosacral angle values ranged from $49^{\circ}$ to $87^{\circ}$ (mean $69.8^{\circ}$ ) and were consistent with severe lumbosacral 
TABLE 1. Details of preoperative data in 15 patients with HDDS*

\begin{tabular}{ccccc}
\hline Variable & Minimum & Maximum & Mean & SD \\
\hline PI & 59 & 96 & 74.53 & 10.93 \\
\hline PT & 12 & 47 & 30.67 & 10.84 \\
\hline SS & 17 & 66 & 44.87 & 13.34 \\
\hline LSA & 49 & 87 & 69.80 & 12.66 \\
\hline TK & -16 & 23 & 9.27 & 9.04 \\
\hline LL & 40 & 110 & 68.80 & 17.85 \\
\hline
\end{tabular}

$\mathrm{LL}=\mathrm{L} 1-5$ lumbar lordosis; $\mathrm{LSA}=$ lumbosacral angle; $\mathrm{PI}=$ pelvic incidence; $\mathrm{PT}$ = pelvic tilt; $\mathrm{SS}=$ sacral slope; $\mathrm{TK}=\mathrm{T4}-12$ thoracic kyphosis.

* All values are degrees.

kyphosis for all patients. The values of preoperative sagittal angular parameters are reported in Table 1 . The same angular values were taken 6 months postoperatively and are reported in Table 2. At a mean follow-up of 3.8 years (range 2-7 years), the same angular values were noted and are reported in Table 3.

Neurological complications were noted in 5 patients. An incomplete (motor strength 2/5) unilateral L-5 motor and sensitivity deficit occurred in 3 patients, and an incomplete unilateral L-5 (motor strength 2/5) and S-1 motor (motor strength $3 / 5$ ) and sensitivity deficit occurred in 2 patients. At final follow-up (2-7 years), L4-S1 fusion was assessed based on the absence of mobility on lateral dynamic flexion-extension radiographs. No patient had persistent neurological deficit or radicular pain. Full neurological recovery was obtained between 2 and 18 months postoperatively. Postoperative complications included 5 instances of superficial wound dehiscence, requiring iterative closure and local care in 3 patients. Thirteen of the 15 patients had no functional limitation and no pain at final follow-up. Two patients had mild and occasional low-back pain, which presented after a day of sports or work.

Regarding the postoperative severity of spondylolisthesis, all 15 patients were classified as having a Grade 1 Meyerding slip $(<25 \%)$ at final follow-up (mean anterior residual slip of L-5 was $17 \%$ ).

Statistical comparisons between the different angular values were made using a Wilcoxon's rank test and showed no significant differences between preoperative, 6-month, and final follow-up values of pelvic incidence, pelvic tilt, and sacral slope. Statistical analysis showed a significant increase in 6-month and final follow-up lumbosacral angle values compared with preoperative values $(p<0.001)$ (Fig. 2). A significant increase in thoracic kyphosis was

TABLE 2. Details of angular parameters at 6 months in 15 patients with HDDS*

\begin{tabular}{lrrrc}
\hline Value & Minimum & Maximum & Mean & SD \\
\hline PI & 58 & 93 & 73.20 & 9.65 \\
\hline PT & 18 & 37 & 25.53 & 4.79 \\
\hline SS & 30 & 60 & 45.80 & 7.61 \\
\hline LSA & 96 & 113 & 104.47 & 5.62 \\
\hline TK & -13 & 31 & 15.13 & 9.98 \\
\hline LL & 26 & 68 & 46.53 & 12.50 \\
\hline
\end{tabular}

* All values are degrees.
TABLE 3. Details of angular parameters in 15 patients with HDDS at final follow-up*

\begin{tabular}{ccccc}
\hline Variable & Minimum & Maximum & Mean & SD \\
\hline PI & 62 & 92 & 76.93 & 9.21 \\
\hline PT & 10 & 37 & 25.07 & 6.51 \\
\hline SS & 35 & 56 & 46.47 & 7.20 \\
\hline LSA & 96 & 120 & 105.13 & 6.97 \\
\hline TK & 8 & 38 & 25.67 & 8.10 \\
\hline LL & 35 & 56 & 45.93 & 5.19 \\
\hline
\end{tabular}

* All values are degrees.

noted between the preoperative and 6-month radiographs $(\mathrm{p}<0.001)$, which increased again between the 6-month and final follow-up radiographs $(\mathrm{p}<0.001)$ (Fig. 3). A statistically significant decrease in lumbar lordosis was noted between the preoperative and 6-month radiographs ( $\mathrm{p}<$ $0.001)$ and between the preoperative and follow-up radiographs $(\mathrm{p}<0.001)$ (Fig. 4). The details of statistical study are reported in Table 4.

\section{Discussion}

During the past decade, many studies have reported relationships between spinopelvic morphology and spondylolisthesis. Duval-Beaupère and colleagues ${ }^{5,15}$ described the pelvic incidence as a specific and constant pelvic anatomical parameter for each individual, which determines the orientation of the pelvis. In previous studies, we showed that pelvic incidence differed between healthy subjects and patients with HDDS, and that the observed difference tends to increase in a direct linear fashion as the severity of spondylolisthesis increases. ${ }^{24,26,28}$

In HDDS, local deformity affects the overall posture of the patient, with pelvic retroversion causing flexion of the hip and knee joints and lumbosacral kyphosis leading to compensatory hyperlordosis of the adjacent lum-

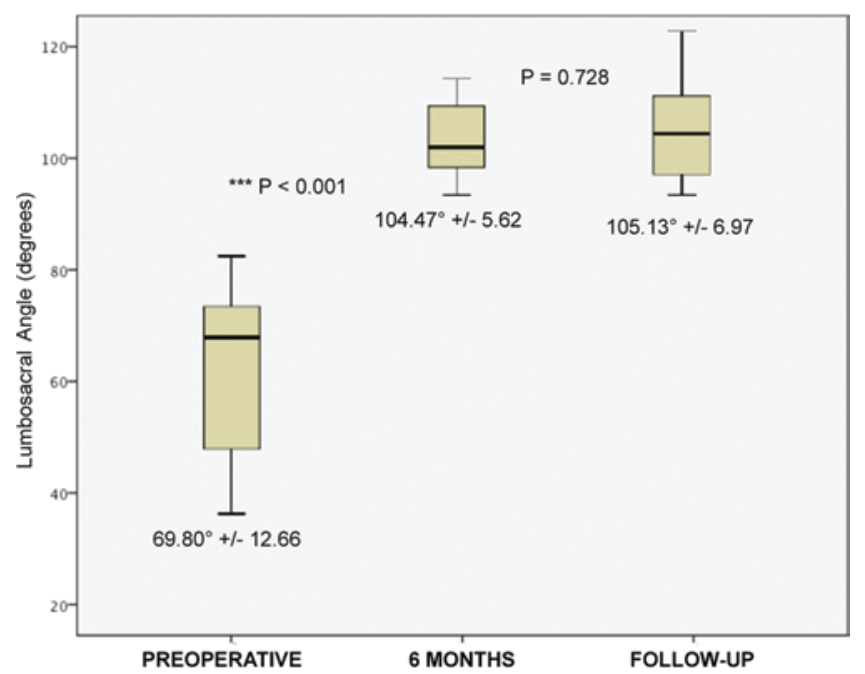

FIG. 2. Schematic representation of lumbosacral angle variation between preoperative, 6 -month postoperative, and follow-up values (Wilcoxon nonparametric test with $p=0.05$ as significance threshold). Figure is available in color online only. 


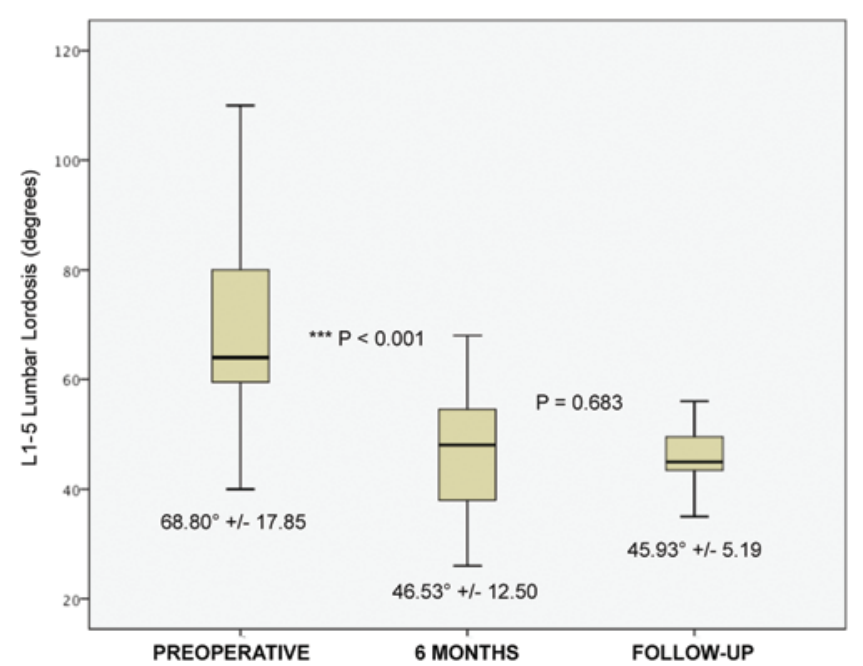

FIG. 3. Schematic representation of lumbar lordosis variation between preoperative, 6-month postoperative, and follow-up values (Wilcoxon nonparametric test with $p=0.05$ as significance threshold). Figure is available in color online only.

bar segments and hypokyphosis in the thoracic area. ${ }^{23}$ In a previous study, we demonstrated deviations in the related measures of sagittal spinopelvic alignment (sacral slope, pelvic tilt, lumbar lordosis, and thoracic kyphosis) in adolescents with HDDS. These subjects stand with an increased sacral slope, pelvic tilt, and lumbar lordosis, but with a decreased thoracic kyphosis. Such sacral slope, pelvic tilt, and lumbar lordosis modifications are compensatory phenomena due to pelvic incidence increase and lumbosacral kyphosis in spondylolisthesis patients. ${ }^{28}$ We demonstrated the same sagittal balance strategy in spondylolisthesis patients as in a control group, regarding negative correlations between pelvic incidence and sacral slope and between pelvic tilt and lumbar lordosis. Contrary to Labelle et al., ${ }^{12,13}$ we found that the decrease in thoracic kyphosis in spondylolisthesis patients was

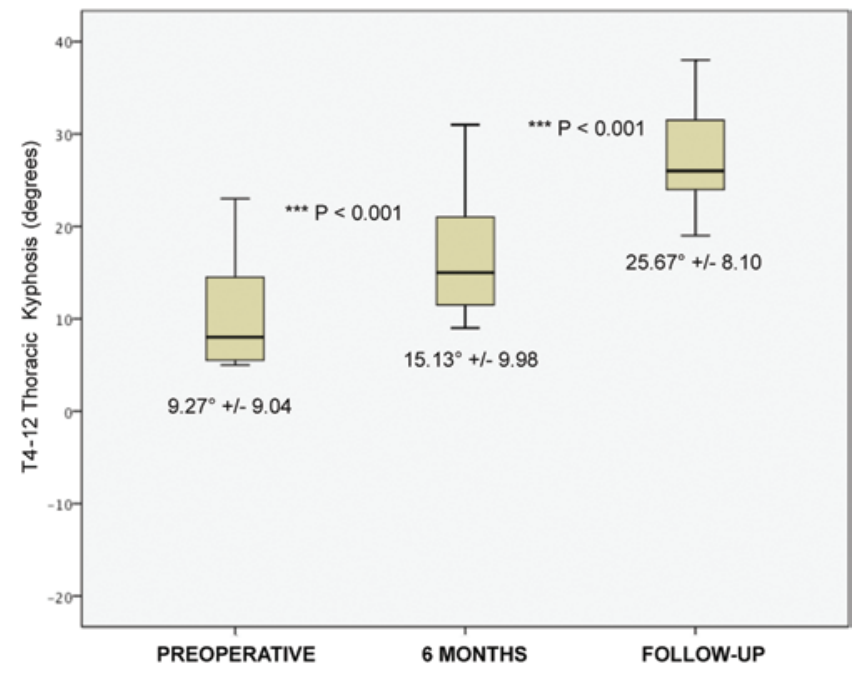

FIG. 4. Schematic representation of thoracic kyphosis variation between preoperative, 6-month postoperative, and follow-up values (Wilcoxon nonparametric test with $p=0.05$ as significance threshold). Figure is available in color online only. not a logical compensatory phenomenon due to lumbar lordosis increase. Our explanation of these thoracic kyphosis values is that, because of anterior trunk displacement associated with L-5 anterior slip, thoracic kyphosis decreases to avoid displacing the gravity line of the trunk anteriorly to the femoral heads. ${ }^{28}$

Ideally, surgery in patients with HDDS should restore normal anatomy with minimal functional restriction. The goals of such treatment should be to relieve pain, to prevent slip progression, and to improve function. All of these goals may be achieved, in theory, by a solid fusion and a complete correction of sagittal trunk imbalance. For this reason, we designed a surgical strategy that combines reduction of lumbosacral kyphosis with anterior fusion via a posterior approach. Correction of lumbosacral kyphosis was very good compared with other published data in the literature, ${ }^{2}$ and the fusion rate in our series proved to be optimal. In our experience, this technique produced both an effective reduction of L-5 slip (from 73\% to 17\%) and a significant reduction of lumbosacral kyphosis (from $69.8^{\circ}$ to $105.13^{\circ}$ ). The use of L4-5 anterior interbody support and L5-S1 bone graft provided strong and stable fusion in all cases. This technique is currently our preferred treatment method for patients with significant lumbopelvic imbalance.

In the current study, we demonstrated that correction of pelvic retroversion and lumbosacral kyphosis had an immediate impact on pelvic alignment (lumbosacral kyphosis), as well as a progressive but major impact on lumbar lordosis and thoracic kyphosis above (Fig. 5). Due to complete reduction of lumbosacral kyphosis and anterior trunk displacement associated with L-5 anterior slip, lumbar lordosis progressively decreased and thoracic kyphosis progressively increased postoperatively. The impact on the lumbopelvic morphology after surgical correction increased proportionally to the degree of lumbosacral kyphosis deformity. Adjusting the sagittal balance of the trunk produced not only pelvic anteversion, but also reciprocal adjustment of lumbar lordosis and thoracic kyphosis, up to a normal and compensated level of global sagittal balance.

Unlike evaluation of patients with surgically treatable scoliosis, which requires careful analysis of flexibility radiographs to predict the degree of spontaneous correction of adjacent curves, the potential postoperative sagittal improvement of HDDS patients remains difficult to appreciate due to hamstring and spine stiffness..$^{24,25}$ However, our impression regarding our small patient cohort is this: the younger the patient, the faster the improvement in lumbar lordosis and thoracic kyphosis values. Further investigations are necessary to determine whether these compensatory phenomena could also be found in HDDS patients treated after skeletal maturity. ${ }^{22}$

Although in situ fusion is an effective treatment for lower grades of pediatric spondylolisthesis, studies have reported that this technique results in a high rate of pseudarthrosis, slip progression, and persistent cosmetic deformity in HDDS patients. ${ }^{2,20}$ In patients with HDDS treated using in situ fusion, adjacent mobile segments in the upper lumbar spine, pelvis orientation, and thoracic spine compensate for the local sagittal imbalance of the lumbosacral 
TABLE 4. Details of statistical comparisons between preoperative, 6-month, and follow-up angular parameters

\begin{tabular}{|c|c|c|c|c|c|c|}
\hline \multirow[b]{2}{*}{ Variable } & \multicolumn{3}{|c|}{ Mean $\pm \mathrm{SD}\left({ }^{\circ}\right)$} & \multicolumn{3}{|c|}{ p Value ${ }^{*}$} \\
\hline & Preop & 6 Mos & Follow-Up & $\begin{array}{c}\text { Preop vs } 6 \\
\text { Mos }\end{array}$ & $\begin{array}{l}6 \text { Mos vs } \\
\text { Follow-Up }\end{array}$ & $\begin{array}{l}\text { Preop vs } \\
\text { Follow-Up }\end{array}$ \\
\hline $\mathrm{PI}$ & $74.53 \pm 10.93$ & $73.20 \pm 9.65$ & $76.93 \pm 9.21$ & 0.137 & 0.066 & 0.549 \\
\hline PT & $30.67 \pm 10.84$ & $25.53 \pm 4.79$ & $25.07 \pm 6.51$ & 0.068 & 0.647 & 0.078 \\
\hline SS & $44.87 \pm 13.34$ & $45.80 \pm 7.61$ & $46.47 \pm 7.20$ & 0.798 & 0.820 & 0.730 \\
\hline LSA & $69.80 \pm 12.66$ & $104.47 \pm 5.62$ & $105.13 \pm 6.97$ & 0.001 & 0.728 & 0.001 \\
\hline TK & $9.27 \pm 9.04$ & $15.13 \pm 9.98$ & $25.67 \pm 8.10$ & 0.001 & 0.001 & 0.001 \\
\hline $\mathrm{LL}$ & $68.80 \pm 17.85$ & $46.53 \pm 12.5$ & $45.93 \pm 5.19$ & 0.001 & 0.683 & 0.001 \\
\hline
\end{tabular}

* Wilcoxon rank test. Values in boldface are statistically significant.

junction. The result is a suboptimal, but satisfactory global sagittal balance of the trunk, even in the most severe grade of slipping. ${ }^{28}$

We recognize that this study presents several shortcomings. First, this small group of patients has some inherent selection bias present. Nonetheless, this is a homogeneous group of patients with similar preoperative deformities and demographic differences, all treated by a single surgeon. Another potential criticism is that our postoperative evaluation was not uniform, especially regarding patient ages and the timing of the final follow-up. The variations in final evaluations preclude us from defining the exact kinetics and the optimal age or skeletal maturity regarding spontaneous sagittal modifications in our cohort. Finally, we did not use any global criteria to appreciate sagittal balance, such as the C-7 plumb line or T-1 sagittal offset; we only stressed the importance of an improved lumbar lordosis/thoracic kyphosis ratio with a good clinical standing posture in our patients.

Regarding mean values of pelvic incidence, pelvic tilt,
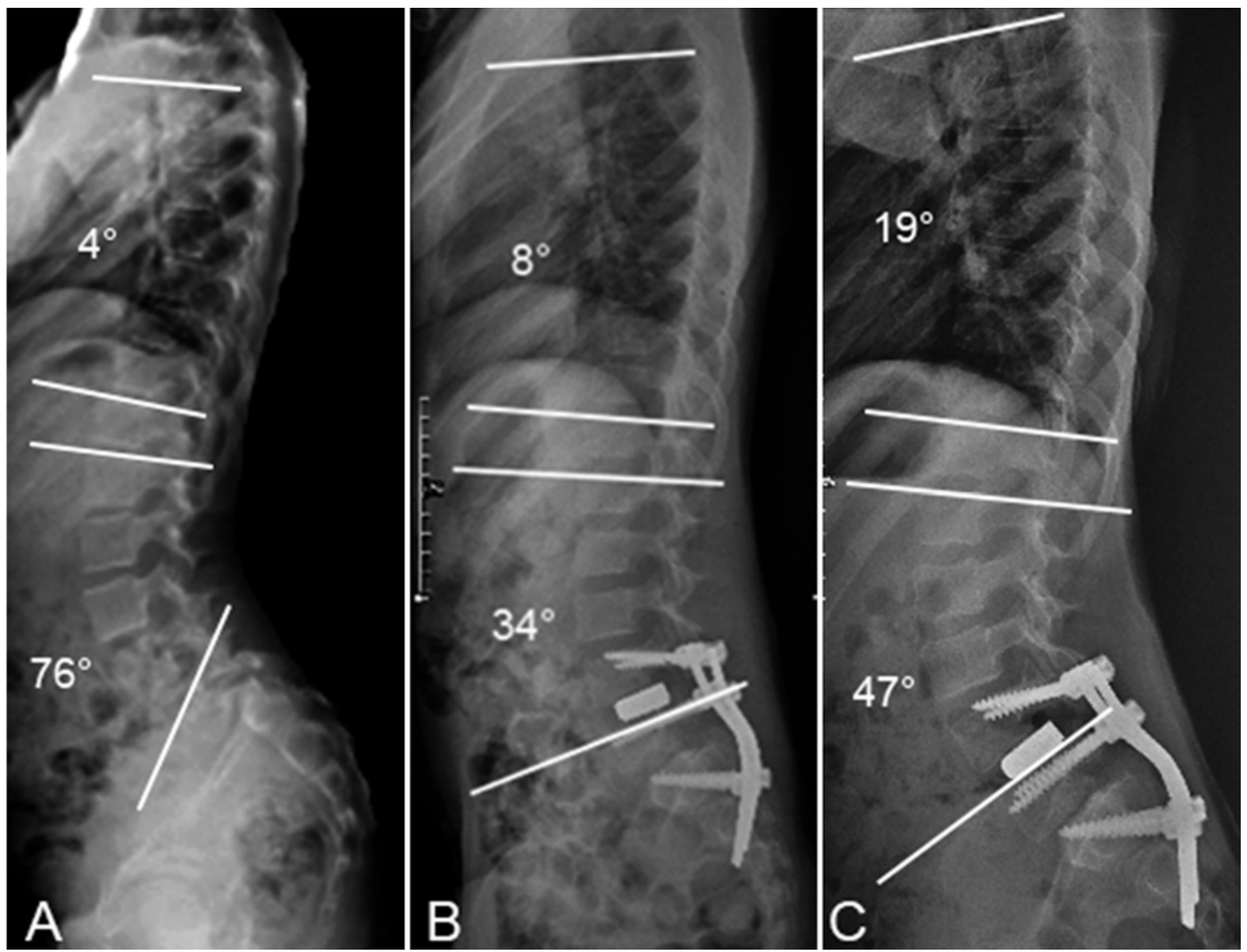

FIG. 5. Preoperative (A), 6-month postoperative (B), and follow-up (C) lateral full-spine standing radiographs obtained in a 10.5-year-old patient with HDDS. Note the complete correction of lumbosacral kyphosis and progressive reciprocal improvement of lumbar lordosis and thoracic kyphosis above. 
and sacral slope, we found 6-month and final follow-up discrepancies, compared with the expected findings of pelvic incidence $=$ pelvic tilt + sacral slope. In high-grade spondylolisthesis, severe dystrophic changes of the upper sacral endplate may cause technical difficulties in pelvic incidence and sacral slope measurement. ${ }^{27}$ Additionally, the placement of intrasacral rods and screws could modify the position of anatomical landmarks. We also noted small differences in angular measurements between preoperative, 6-month, and final follow-up pelvic incidence values. In the same manner, we explain small variations between mean sacral slope and pelvic tilt values and deviations from the normal formula pelvic incidence $=$ pelvic tilt + sacral slope regarding main values. However, case-by-case analysis regarding this formula is consistent with maintained reciprocal values, and pelvic incidence $=$ pelvic tilt + sacral slope on preoperative, 6-month, and final followup radiographs in our patients.

\section{Conclusions}

We postulate that our surgical strategy entails the most complete correction of the local deformity with the shortest possible fusion. Improved reduction of L-5 slippage and complete correction of lumbosacral kyphosis allow for progressive normalization of the overall sagittal profile. The load distribution in the adjacent segments is normalized, thus avoiding potential degeneration of adjacent discs. ${ }^{1,9}$ The potential benefits of restoring sagittal spinal balance must be weighed against the risks of reduction. In the case of high-grade slips, the risk of neurological deficit is the principal concern. Review of the neurological complications reported in published cases in which patients underwent substantial reduction and fusion for HDDS appear to compare favorably with those reported using other techniques. ${ }^{2}$ The procedure is technically demanding and should be performed by spinal surgeons who are experienced in performing successful anterior and posterior fusions.

\section{References}

1. Barrey C, Jund J, Noseda O, Roussouly P: Sagittal balance of the pelvis-spine complex and lumbar degenerative diseases. A comparative study about 85 cases. Eur Spine J 16:14591467,2007

2. Bouyer B, Bachy M, Courvoisier A, Dromzee E, Mary P, Vialle R: High-grade lumbosacral spondylolisthesis reduction and fusion in children using transsacral rod fixation. Childs Nerv Syst 30:505-513, 2014

3. Boxall D, Bradford DS, Winter RB, Moe JH: Management of severe spondylolisthesis in children and adolescents. J Bone Joint Surg Am 61:479-495, 1979

4. During J, Goudfrooij H, Keessen W, Beeker TW, Crowe A: Toward standards for posture. Postural characteristics of the lower back system in normal and pathologic conditions. Spine (Phila Pa 1976) 10:83-87, 1985

5. Duval-Beaupère G, Schmidt C, Cosson P: A Barycentremetric study of the sagittal shape of spine and pelvis: the conditions required for an economic standing position. Ann Biomed Eng 20:451-462, 1992

6. Funao H, Tsuji T, Hosogane N, Watanabe K, Ishii K, Nakamura M, et al: Comparative study of spinopelvic sagittal alignment between patients with and without degenerative spondylolisthesis. Eur Spine J 21:2181-2187, 2012

7. Gelb DE, Lenke LG, Bridwell KH, Blanke K, McEnery KW: An analysis of sagittal spinal alignment in 100 asymptomatic middle and older aged volunteers. Spine (Phila Pa 1976) 20:1351-1358, 1995

8. Hresko MT, Hirschfeld R, Buerk AA, Zurakowski D: The effect of reduction and instrumentation of spondylolisthesis on spinopelvic sagittal alignment. J Pediatr Orthop 29:157162,2009

9. Kim KH, Lee SH, Shim CS, Lee DY, Park HS, Pan WJ, et al: Adjacent segment disease after interbody fusion and pedicle screw fixations for isolated L4-L5 spondylolisthesis: a minimum five-year follow-up. Spine (Phila Pa 1976) 35:625634,2010

10. Kim MK, Lee SH, Kim ES, Eoh W, Chung SS, Lee CS: The impact of sagittal balance on clinical results after posterior interbody fusion for patients with degenerative spondylolisthesis: a pilot study. BMC Musculoskelet Disord 12:69, 2011

11. Labelle H, Mac-Thiong JM, Roussouly P: Spino-pelvic sagittal balance of spondylolisthesis: a review and classification. Eur Spine J 20 (Suppl 5):641-646, 2011

12. Labelle H, Roussouly P, Berthonnaud E, Dimnet J, O'Brien M: The importance of spino-pelvic balance in L5-S1 developmental spondylolisthesis: a review of pertinent radiologic measurements. Spine (Phila Pa 1976) 30 (6 Suppl):S27S34, 2005

13. Labelle H, Roussouly P, Berthonnaud E, Transfeldt E, O'Brien M, Chopin D, et al: Spondylolisthesis, pelvic incidence, and spinopelvic balance: a correlation study. Spine (Phila Pa 1976) 29:2049-2054, 2004

14. Labelle H, Roussouly P, Chopin D, Berthonnaud E, Hresko T, O'Brien M: Spino-pelvic alignment after surgical correction for developmental spondylolisthesis. Eur Spine J 17:11701176,2008

15. Legaye J, Duval-Beaupère G, Hecquet J, Marty C: Pelvic incidence: a fundamental pelvic parameter for three-dimensional regulation of spinal sagittal curves. Eur Spine J 7:99-103, 1998

16. Li Y, Hresko MT: Radiographic analysis of spondylolisthesis and sagittal spinopelvic deformity. J Am Acad Orthop Surg 20:194-205, 2012

17. Lim JK, Kim SM: Difference of sagittal spinopelvic alignments between degenerative spondylolisthesis and isthmic spondylolisthesis. J Korean Neurosurg Soc 53:96-101, 2013

18. Mac-Thiong JM, Labelle H: A proposal for a surgical classification of pediatric lumbosacral spondylolisthesis based on current literature. Eur Spine J 15:1425-1435, 2006

19. Mac-Thiong JM, Wang Z, de Guise JA, Labelle H: Postural model of sagittal spino-pelvic alignment and its relevance for lumbosacral developmental spondylolisthesis. Spine (Phila Pa 1976) 33:2316-2325, 2008

20. Martiniani M, Lamartina C, Specchia N: "In situ" fusion or reduction in high-grade high dysplastic developmental spondylolisthesis (HDSS). Eur Spine J 21 (Suppl 1):S134-S140, 2012

21. Meyerding HW: Spondylolisthesis; surgical fusion of lumbosacral portion of spinal column and interarticular facets; use of autogenous bone grafts for relief of disabling backache. $\mathbf{J}$ Int Coll Surg 26:566-591, 1956

22. Park SJ, Lee CS, Chung SS, Kang KC, Shin SK: Postoperative changes in pelvic parameters and sagittal balance in adult isthmic spondylolisthesis. Neurosurgery 68 (2 Suppl Operative):355-363, 2011

23. Roussouly P, Pinheiro-Franco JL: Biomechanical analysis of the spino-pelvic organization and adaptation in pathology. Eur Spine J 20 (Suppl 5):609-618, 2011 
24. Vialle R, Benoist M: High-grade lumbosacral spondylolisthesis in children and adolescents: pathogenesis, morphological analysis, and therapeutic strategy. Joint Bone Spine 74:414417, 2007

25. Vialle R, Charosky S, Padovani JP, Rigault P, Glorion C: Surgical treatment of high-grade lumbosacral spondylolisthesis in childhood, adolescent and young adult by the "doubleplate" technique: a past experience. Eur Spine J 15:12101218,2006

26. Vialle R, Dauzac C, Khouri N, Wicart P, Glorion C, Guigui $\mathrm{P}$ : Sacral and lumbar-pelvic morphology in high-grade spondylolisthesis. Orthopedics 30:642-649, 2007

27. Vialle R, Ilharreborde B, Dauzac C, Guigui P: Intra and inter-observer reliability of determining degree of pelvic incidence in high-grade spondylolisthesis using a computer assisted method. Eur Spine J 15:1449-1453, 2006

28. Vialle R, Ilharreborde B, Dauzac C, Lenoir T, Rillardon L, Guigui P: Is there a sagittal imbalance of the spine in isthmic spondylolisthesis? A correlation study. Eur Spine J 16:16411649, 2007

29. Vialle R, Levassor N, Rillardon L, Templier A, Skalli W,
Guigui P: Radiographic analysis of the sagittal alignment and balance of the spine in asymptomatic subjects. J Bone Joint Surg Am 87:260-267, 2005

\section{Author Contributions}

Conception and design: Vialle, Thomas. Acquisition of data: Vialle, Thomas, Bachy, Dubory. Analysis and interpretation of data: Vialle, Thomas, Bachy. Drafting the article: Vialle, Bachy, Courvoisier, Dubory. Critically revising the article: Vialle, Courvoisier, Bouloussa. Reviewed submitted version of manuscript: Vialle, Courvoisier, Bouloussa. Approved the final version of the manuscript on behalf of all authors: Vialle. Statistical analysis: Vialle, Dubory, Bouloussa. Study supervision: Vialle.

\section{Correspondence}

Raphaël Vialle, Armand Trousseau Hospital, Department of Paediatric Orthopaedics, 26 avenue du Docteur Arnold Netter, 75571 Paris Cedex 12, France. email: raphael.vialle@trs.aphp.fr. 